\title{
UN ASSEMBLAGE DE PETITS VERTÉBRÉS HAUTEMENT DIVERSIFIÉ DE LA FIN DU MIS 5 DANS UN ENVIRONNEMENT MONTAGNARD AU CENTRE DE L'ESPAGNE (CUEVA DEL CAMINO, PINILLA DEL VALLE, COMMUNAUTÉ AUTONOME DE MADRID)
}

\author{
César LAPLANA ${ }^{1}$, Hugues-Alexandre BLAIN ${ }^{2,3}$, Paloma SEVILLA ${ }^{4}$, \\ Juan Luis ARSUAGA ${ }^{4,5}$, Enrique BAQUEDANO ${ }^{1}$ \& Alfredo PÉREZ-GONZÁLEZ ${ }^{6}$
}

\begin{abstract}
RÉSUMÉ
La Haute Vallée du Lozoya au centre de l'Espagne est actuellement l'un des environnements naturels le plus diversifié en ce qui concerne les petits vertébrés terrestres dans la péninsule Ibérique, avec 13 espèces d'amphibiens, 17 de reptiles et 26 de micromammifères. Les phénomènes de karstification des calcaires crétacés qui affleurent de chaque côté de la vallée du Lozoya ont formé des cavités, dont la plupart préservent des sédiments d'âge pléistocène supérieur et holocène contenant d'abondants restes fossiles ainsi que des ossements humains néanderthaliens actuellement en cours d'étude. Le premier de ces sites à avoir été découvert est celui de Cueva del Camino. Cette cavité, aujourd'hui démantelée, a conservé des sédiments du Pléistocène supérieur ancien datés d'entre $91,6 \pm 8,1 \mathrm{ka}$ et 74,5 $\pm 6,3 \mathrm{ka}$ et a préservé les évidences de son utilisation comme repaire par les hyènes. En plus des fossiles de grands mammifères, probablement introduits dans la cavité par les hyènes, au moins 49 espèces de petits vertébrés ( 7 amphibiens, 11 reptiles et 31 micromammifères) sont représentées dans cet assemblage, indiquant qu'à cette époque, la Haute Vallée du Lozoya hébergeait déjà une communauté de petits vertébrés hautement diversifiée, aussi riche voire peut-être même plus riche qu'actuellement. Les conditions ayant pu favoriser cette haute richesse spécifique sont analysées.
\end{abstract}

Mots-clés : petits vertébrés, biodiversité, paléoclimatologie, paléoécologie, Pléistocène supérieur, MIS 5, Espagne

\section{ABSTRACT}

A HIGHLY DIVERSIFIED SMALL VERTEBRATE ASSEMBLAGE FROM MIS 5 IN A MOUNTAIN ENVIRONMENT IN CENTRAL SPAIN (CUEVA DEL CAMINO, PINILLA DEL VALLE, AUTONOMOUS REGION OF MADRID)

The High Valley of the Lozoya in Central Spain is presently one of the most diversified environments for small terrestrial vertebrates in the Iberian Peninsula, with 13 species of amphibians, 17 reptiles and 26 small mammals. Karstification of the Cretaceous limestones that crop out on either slopes of the Lozoya Valley has given place to the formation of caves, many of which preserve Upper Pleistocene and Holocene sediments with abundant fossils and human remains, currently under study. The first of these fossi localities to be discovered was the Cueva del Camino, presently a dismantled cave that preserves sediments dated between $91.6 \pm 8.1$ $\mathrm{ka}$ and $74.5 \pm 6.3 \mathrm{ka}$, containing evidence of having been used as a den by hyenas. Besides large mammal fossils, probably introduced in the cave by hyenas, at least 49 species of small vertebrates ( 7 amphibians, 11 reptiles and 31 small mammals) are represented in this assemblage, indicating that at that time, the Lozoya Valley had a highly diversified community of small vertebrates, equally rich or even richer than today. The conditions favouring this high species richness are analysed.

Keywords: small vertebrates, biodiversity, paleoclimatology, paleoecology, Late Pleistocene, MIS 5, Spain

\section{1 - INTRODUCTION}

La connaissance du Stade Isotopique Marin 5 (MIS 5) ou Dernier Interglaciaire sensu lato présente un intérêt certain puisqu'il s'agit de l'analogue le plus récent du climat actuel. Les changements climatiques qui se sont produits lors de cette période et les modifications occasionnées dans la distribution de la flore et de la faune

\footnotetext{
${ }^{1}$ Museo Arqueológico Regional de Madrid, Plaza de las Bernardas s/n, E-28801, ALCALÁ DE HENARES.

Courriels : cesar.laplana.conesa@madrid.org; enrique.baquedano@madrid.org

${ }^{2}$ IPHES, Institut Català de Paleoecologia Humana i Evolució Social, C/ Escorxador s/n, E-43003 TARRAGONE. Courriel: hablain@iphes.cat

${ }^{3}$ Area de Prehistoria, Universitat Rovira i Virgili (URV), Avinguda de Catalunya 35, E-43002 TARRAGONE.

${ }^{4}$ Departamento de Paleontología, Facultad de Ciencias Geológicas (UCM) C/ Jose Antonio Novais 2, E-28040 MADRID.

Courriels: psevilla@geo.ucm.es;jlarsuaga@isciii.es

${ }^{5}$ Centro Mixto UCM-ISCIII de Evolución y Comportamiento Humanos, C/ Sinesio Delgado, 4, E-28029 MADRID.

${ }^{6}$ Centro Nacional de Investigación sobre la Evolución Humana, Paseo de la Sierra de Atapuerca s/n, E-09004 BURGOS

Courriel: alfredo.perez@cenieh.es
} 
peuvent permettre de prévoir quel sera le comportement des écosystèmes actuels face à un changement climatique similaire. Néanmoins, les données sur cette période sont encore très limitées dans certaines zones géographiques. Dans ce travail, nous présentons les associations de petits vertébrés de Cueva del Camino, un site attribué à la deuxième moitié du MIS 5 et qui se trouve au centre de la péninsule Ibérique (fig. 1). Dans cette aire géographique, les gisements d'âge MIS 5 ayant livré des restes de petits vertébrés sont plutôt rares, comme cela est mis en évidence dans les synthèses régionales de Soto et Sesé (1987), Arribas et Jordá (1999) et Sesé et Soto (2000, 2002a,b), ou dans les synthèses d'Aguirre (1989), Sesé et Sevilla (1996) et López-García (2011) plus étendues d'un point de vue géographique. L'unique exception consiste en la récente description par Sesé et al. (2011a,b) dans cette région de deux sites, Hat et Preresa, datés de la fin du MIS 5. Néanmoins, à la différence de
Cueva del Camino, qui se trouve dans un milieu montagnard, ces deux gisements sont situés dans les dépôts de terrasse d'un bassin sédimentaire à plus basse altitude. La situation de Cueva del Camino, dans le cadre d'un système montagneux, confère à ses associations de petits vertébrés des caractéristiques particulières, dont la plus remarquable est sa haute biodiversité.

\section{2 - LE SITE}

Cueva del Camino est une ancienne grotte («cueva») aujourd'hui en partie démantelée par l'érosion et par les travaux de construction du chemin («camino») qui lui donne son nom. Elle se trouve dans la Haute Vallée du Lozoya, unité physiographique bien définie à l'intérieur du Système Central, une cordillère montagneuse située au centre de la péninsule Ibérique. Le gisement

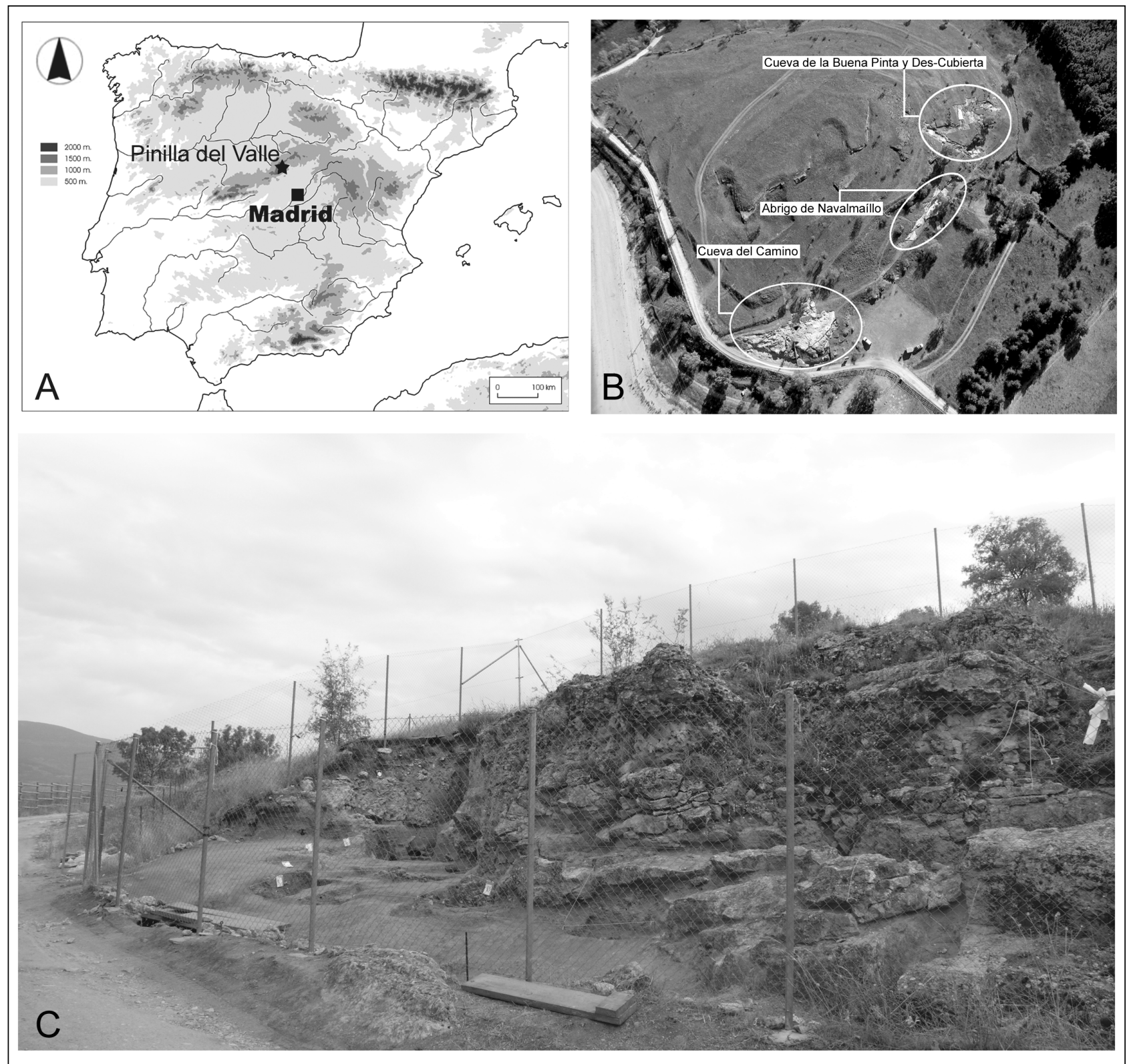

Fig. 1: Localisation géographique (A), vue aérienne du Calvero de la Higuera (B) et photographie générale (C) de Cueva del Camino (Pinilladel-Valle, Madrid).

Fig. 1: Geographical location (A), aerial view of the Calvero de la Higuera (B) and general view (C) of Cueva del Camino (Pinilla-del-Valle, Madrid). 
se situe à proximité du fond de la vallée, à une altitude de $1095 \mathrm{~m}$, tandis que les reliefs qui l'entourent atteignent des altitudes avoisinant les $1800 \mathrm{~m} \mathrm{au}$ sud-est et les $2000 \mathrm{~m}$ au nord-est, jusqu'à $2430 \mathrm{~m}$ pour les sommets les plus élevés. Dans les montagnes environnantes, sont visibles quelques-uns des témoins de phénomènes glaciaires les plus méridionaux d'Europe (Palacios et al., 2012). La faune et la flore actuelle de la Haute Vallée du Lozoya présentent un grand intérêt biogéographique, avec des espèces de haute montagne, nordiques et des espèces d'affinité eurosibérienne, qui trouvent ici leur limite de distribution méridionale, en même temps que d'autres espèces avec des affinités plus méditerranéennes (Prieto Cana $\&$ de Lucio, 1995). Tout cela implique que la diversité de cette région soit particulièrement élevée par rapport à d'autres zones du centre péninsulaire.

Le gisement de Cueva del Camino fait partie de l'ensemble de remplissages karstiques du Calvero de la Higuera (fig. 1). Ces gisements sont situés sur la commune de Pinilla del Valle (Madrid) dans la Haute Vallée du Lozoya. Cueva del Camino fut le premier de ces gisements à être fouillé. Appelé dans un premier temps, comme il apparaît dans les premières publications, "gisement de Pinilla del Valle», il fut découvert en 1979 (Alférez et al., 1982) comme conséquence de la construction d'un chemin sur les bords de la retenue d'eau de la rivière Lozoya. Une équipe de paléontologues de l'Université Complutense de Madrid dirigée par le professeur F. Alférez fouilla ce gisement pendant la décennie des années 1980. C'est durant ces premières campagnes de fouilles que furent notamment découvertes deux molaires humaines assignées alors à des pré-Néanderthaliens (Alférez \& Roldán, 1992). Sur la base de ces premières découvertes, la cavité fut alors interprétée comme un lieu d'occupation humaine (Alférez et al., 1982) mais, postérieurement, Díez (1993) réinterpréta le site comme étant un repaire de hyènes. Ce fut plus tard, en 2002, qu'une équipe pluridisciplinaire formée par des paléontologues, des archéologues et des géologues reprit les fouilles à Cueva del Camino, excavant la presque totalité du gisement. Actuellement les recherches sont dirigées par Juan Luis Arsuaga, Enrique Baquedano et Alfredo Pérez-González.

Le remplissage sédimentaire de Cueva del Camino est composé par deux unités principales: la première, à la base de la séquence, est formée par des sédiments fluviaux (argiles, sables et graviers de roches métamorphiques, niveaux 3 et 4 ); et la seconde, formée par des sédiments au caractère plus autochtone (sables avec galets carbonatés, niveaux 5 à 9 du secteur nord et remplissage des secteurs central et sud). Entre ces deux unités, se trouvent les restes d'une unité intermédiaire très fortement érodée et formée par des spéléothèmes et des brèches osseuses. La configuration de la grotte permet de diviser son remplissage sédimentaire en quatre secteurs appelés nord, centre, sud et diaclase rouge sans continuité évidente due à la présence fréquente de grands blocs de dolomie qui interrompent la stratigraphie (Arsuaga et al., 2012) (fig. 2).
Une série de datations par thermoluminescence sur grains de quartz et sur carbonates indiquent que l'unité inférieure fluviale s'est déposée à la fin du MIS 6, tandis que l'unité détritique supérieure a fourni des dates d'environ 90 ka pour le secteur nord et d'environ 75 ka pour le secteur sud (Arsuaga et al., 2010, 2012; Baquedano et al., 2010; Huguet et al., 2010; Pérez-González et al., 2010) (fig. 2). Bien que les secteurs soient distribués de façon latérale, les dépôts du secteur central chevauchent stratigraphiquement ceux du secteur nord et sont chevauchés par ceux du secteur sud. Par conséquent les dépôts du secteur central possèdent un âge intermédiaire entre 90 et $75 \mathrm{ka}$.

Dix-huit espèces de grands mammifères ont été identifiées, avec une remarquable diversité de carnivores qui comptent dix espèces (Arsuaga et al., 2010, 2012; Huguet et al., 2010). Ces fossiles sont concentrés dans les secteurs nord et centre, alors qu'ils sont très peu représentés dans le secteur sud. L'association est dominée par le daim, suivi du cerf, du cheval et de la hyène tachetée. Les caractéristiques taphonomiques de cette association montrent que celle-ci provient de l'utilisation de la cavité comme repaire par un groupe de hyènes tachetées (Arsuaga et al., 2010, 2012; Baquedano et al., 2010; Huguet et al., 2010).

\section{3 - MÉTHODES}

Après la reprise des fouilles en 2002, un grand effort a été fait concernant la récupération de tous les restes de petits vertébrés fossiles du fait de leur importance pour les données biochronologiques et de leur grande valeur comme indicateurs paléoenvironnementaux et paléoclimatiques.

Dans la zone en cours de fouille du gisement, des carrés d'un mètre carré ont été ouverts et le sédiment a été décapé en tenant compte des couches naturelles. Pour chaque carré et niveau, les échantillons ont été divisés verticalement en intervalles de $10 \mathrm{~cm}$ afin de pouvoir détecter au sein d'une même couche les possibles variations quant à leur contenu en petits vertébrés. Chaque échantillon de sédiment fouillé a alors été séparé du limon par la technique du lavage-tamisage avec l'utilisation d'une tour de tamis de maille décroissante de $2 \mathrm{~mm}$ et $0,5 \mathrm{~mm}$. Occasionnellement un tamis d' $1 \mathrm{~cm}$ a été ajouté pour retenir les fractions plus grossières (graviers ou petites pierres) et ceci afin d'éviter la fragmentation des restes de petits vertébrés lors de cette opération. Tout le lavage-tamisage a été fait durant les campagnes de fouilles. Les refus de tamis résultants ont été laissés au soleil sur des plastiques, puis une fois secs, ils ont été mis dans des sachets en plastiques dûment étiquetés.

Le tri des fossiles s'est fait à la fois durant les campagnes de fouilles et plus tard au laboratoire. Pour la fraction plus grossière, retenue dans les tamis de $2 \mathrm{~mm}$, les fossiles ont été séparés directement à l'œil $\mathrm{nu}$, alors que les résidus entre $2 \mathrm{~mm}$ et $0,5 \mathrm{~mm}$ ont été séparés à l'aide de loupes binoculaires Leica L2 sous un 


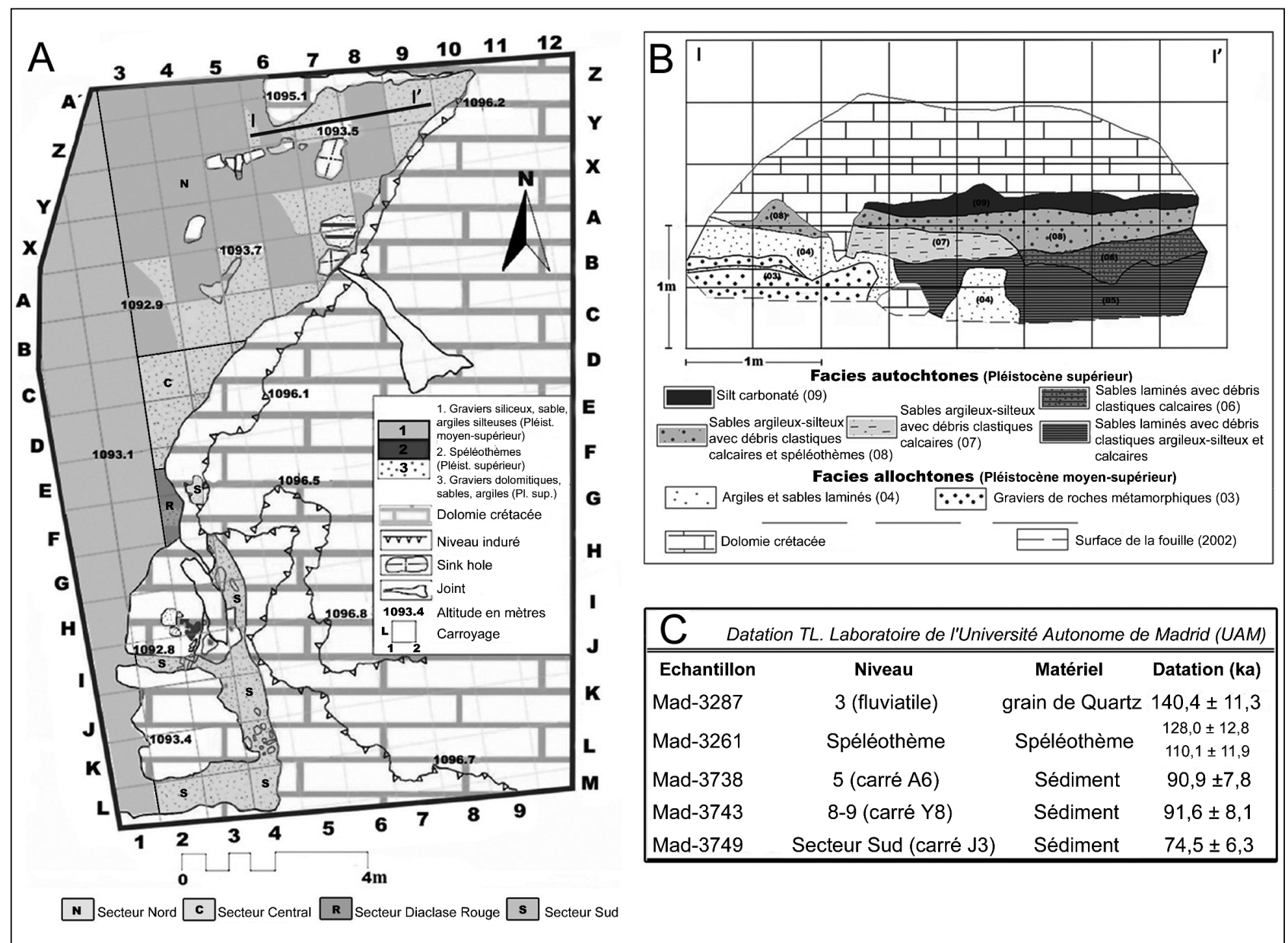

Fig. 2: Plan de la fouille (A), stratigraphie (B) et datations numériques (C) de Cueva del Camino (Pinilla-del-Valle, Madrid), modifié d'après Arsuaga et al. (2012).

Fig. 2: Excavation map (A), stratigraphy (B) and numerical dating (C) of Cueva del Camino (Pinilla-del-Valle, Madrid), modified from Arsuaga et al. (2012).

grossissement de 10X. Tous les ossements et fragments fossiles des échantillons ont été récupérés, même ceux qui ne sont pas identifiables, ainsi que les fragments de charbon, les graines et les divers restes d'invertébrés terrestres.

En plus des restes récupérés par la méthode du lavagetamisage, certains éléments généralement de plus grande taille ont été récupérés directement lors de la fouille, comme cela est le cas pour certaines grandes vertèbres de serpents, quelques robustes humérus de crapauds et aussi et surtout les plaques de chéloniens.

Les petits vertébrés fossiles ont ensuite été étudiés avec une loupe binoculaire Olympus SZX12 pourvue d'une chambre claire et d'un appareil photographique digital Olympus DP71. L'identification ostéologique des ossements et des dents repose principalement sur les travaux de Chaline (1972, 1974), Felten et al. (1973), Szyndlar (1984), Esteban et Sanchiz (1985, 1991), Sevilla (1988), Bailon (1991, 1999), Barahona Quintana (1996), Barahona et Barbadillo (1997) et Blain (2005), entre autres. Les fossiles ont aussi été identifiés à l'aide de matériel de comparaison obtenu à partir de pelotes de réjections, d'excréments de carnivores et d'individus écrasés sur la route récupérés dans le voisinage du gisement, et aussi avec le matériel ostéologique des collections de compa- raison conservé au Museo Nacional de Ciencias Naturales de Madrid (CSIC) et à la Estación Biológica de Doñana (CSIC, Séville).

Les fossiles ont été regroupés en utilisant la méthode du Nombre Minimum d'Individus (NMI). Pour chaque échantillon celui-ci a été déterminé en comptant un élément squelettique diagnostique (les premières molaires inférieures pour les rongeurs) et en prenant en compte, lorsque cela a été possible, la latéralité et le sexe (dans le cas des humérus d'anoures). Afin d'éliminer les biais éventuels liés à l'extension des différents secteurs, nous n'avons utilisé qu'un seul carré suffisamment représentatif pour chaque secteur: Secteur nord: Y8; secteur central : C4; secteur sud: J4.

\section{4 - LES PETITS VERTÉBRÉS}

$\mathrm{Au}$ total, ce sont plusieurs centaines de milliers de restes fossiles de petits vertébrés qui ont ainsi été récupérés à Cueva del Camino depuis la reprise des campagnes de fouilles en 2002. Parmi ces restes ont été déterminés au niveau du genre ou de l'espèce au moins 20000 restes d'amphibiens et de reptiles (représentant plus de 3000 individus), un millier de restes 
d'insectivores et de chiroptères (représentant environ 500 individus) et 9000 dents de rongeurs, représentant environ 700 individus. Dans sa totalité, l'association de petits vertébrés de Cueva del Camino est constituée par 31 espèces de micromammifères (17 rongeurs, 7 insectivores, 5 chiroptères et 2 lagomorphes), 11 reptiles et 7 amphibiens (tab. 1 et 2).

\section{1 - OBSERVATIONS TAPHONOMIQUES}

L'origine de la plus grande partie des restes de micromammifères présents dans les trois secteurs du gisement semble provenir de la prédation, probablement par des carnivores de petite à moyenne taille. Cette interprétation préliminaire se base d'une part, sur le fort pourcentage d'ossements fracturés et digérés et, d'autre part, sur la présence de quelques coprolithes de petite taille contenant des restes de micromammifères. Dans le cas des amphibiens, des reptiles et des chiroptères, connus pour fréquenter les cavités (la zone de pénombre pour les organismes troglophiles comme les chauves-souris ou la zone vestibulaire pour les organismes trogloxènes comme certains amphibiens) durant au moins une partie de l'année, la mort sur place durant l'hivernation est aussi fort probable.

\section{2 - AMPHIBIENS ET REPTILES}

Onze reptiles et sept amphibiens sont documentés à l'état fossile à Cueva del Camino. Tous, à l'exception de la tortue d'Hermann, sont actuellement présents dans la vallée du Lozoya. Quant à la répartition spatiale entre les différents secteurs, les espèces à caractère plus thermophiles (comme Pelobates cultripes, Rhinechis scalaris et surtout Testudo hermanni) sont représentées majoritairement dans le secteur central et surtout dans le secteur nord, où elles totalisent respectivement $6 \%$ et $15,9 \%$ de l'assemblage (fig. 3). Le secteur sud voit une diminution du nombre d'espèces par rapport aux secteurs central et nord, et certaines espèces connues pour être favorisées par les environnements perturbés prédominent largement l'association du secteur sud comme Bufo calamita (78,2 \%) et dans une moindre mesure Alytes cf. obstetricans $(14,3 \%)$ (fig. 3$)$.

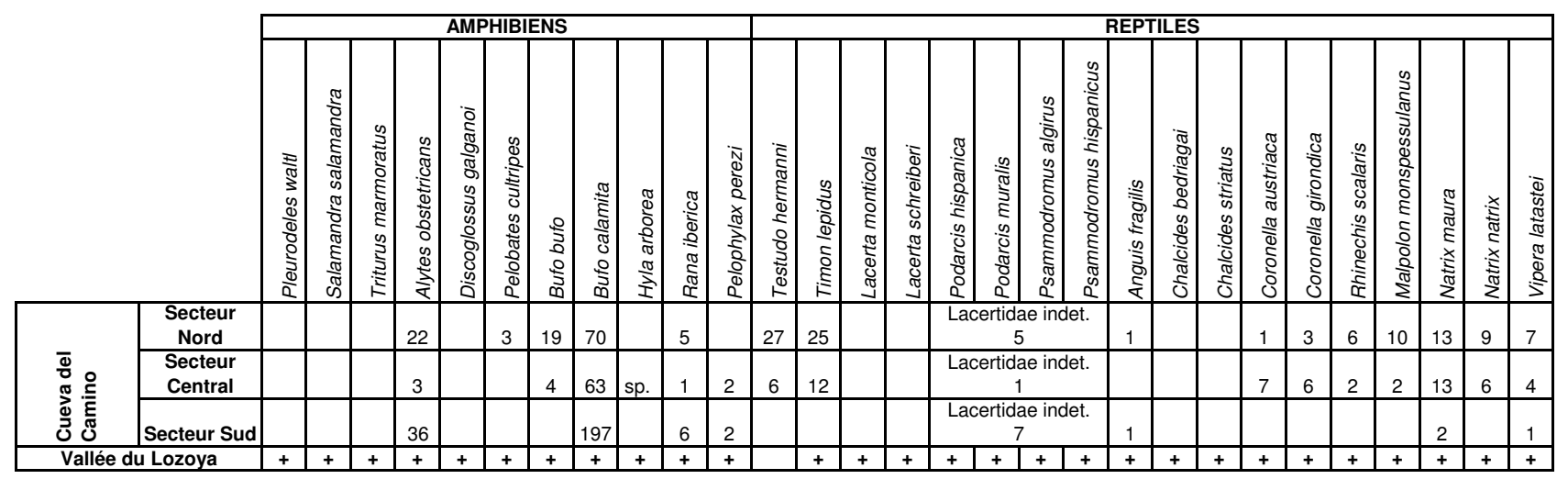

Tab. 1 : Distribution du Nombre Minimum d'Individus (NMI) des espèces d'amphibiens et de reptiles identifiés à Cueva del Camino suivant les secteurs et liste des espèces représentées actuellement dans la Haute Vallée du Lozoya.

Pour chaque secteur, un seul carré représentatif a été utilisé afin d'établir le NMI: secteur nord: Y8; secteur central : C4; secteur sud: J4. Pour Hyla sp., sa présence dans le secteur central n'a pas été établie dans le carré C4 et par conséquent est indiquée ici sans utiliser le NMI.

Tab. 1: Distribution in Minimum Number of Individuals (MNI) of the amphibian and reptile species identified as fossils in each sectors of Cueva del Camino and list of the species that are currently living in the High Valley of the Lozoya river. For each sector, a single representative excavation square has been used to calculate the MNI: sector north: Y8; sector central: C4; sector south: J4. In the case of Hyla sp., its presence in the Sector Central has not been established in the square C4 and consequently is indicated here without using MNI.

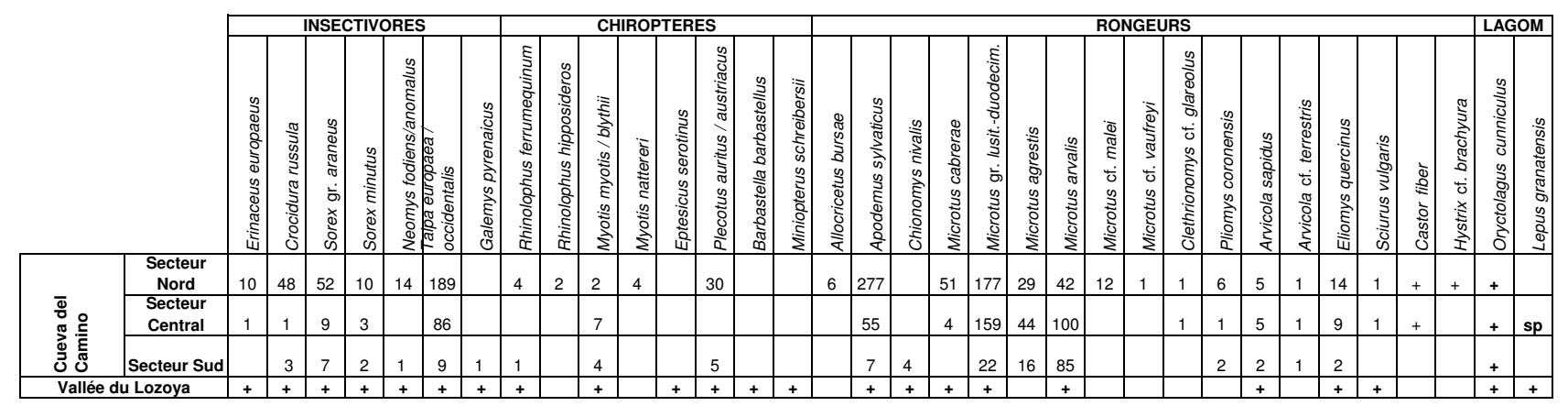

Tab. 2: Distribution par secteur du Nombre Minimum d'Individus (NMI) des espèces fossiles de petits mammifères identifiées à Cueva del Camino et liste des espèces vivant actuellement dans la Haute Vallée du Lozoya.

Pour chaque secteur, un seul carré représentatif a été utilisé afin d'établir le NMI : secteur nord: Y8; secteur central : C4; secteur sud: J4. Dans le cas des rongeurs, le NMI a été calculé selon le secteur à partir des premières molaires inférieures, ce qui explique l'absence dans cette figure de taxons comme Castor et Hystrix qui ne sont pas représentés par cet élément.

Tab. 2: Distribution per sector of the Minimum Number of Individuals (MNI) of fossil small mammal species identified at Cueva del Camino and list of the species presently living in the High Valley of the Lozoya river. For each sector, a single representative excavation square has been used to calculate the MNI: sector north: Y8; sector central: C4; sector south: J4. In the case of rodents, the minimum number of individuals has been calculated for each sector only taking into account the first lower molars, which explains the absence of taxa like Castor and Hystrix in this figure since they are not represented by this element in the assemblages. 


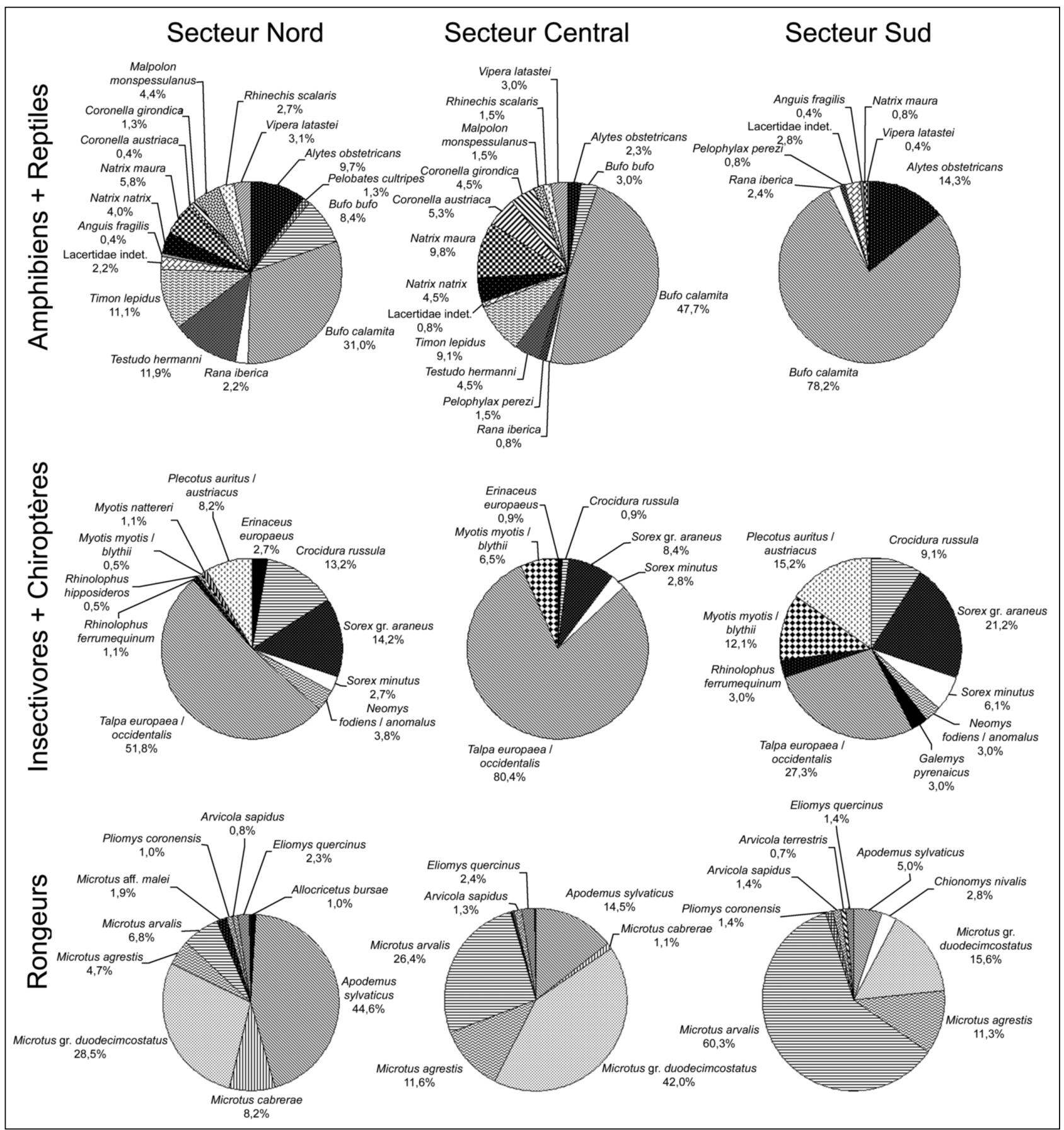

Fig. 3 : Représentation en pourcentage et par secteur du nombre minimum d'individus (NMI) des taxons identifiés à Cueva del Camino.

Afin d'éliminer les biais éventuels résultant de l'extension inégale des différents secteurs, nous n'avons utilisé qu'un seul carré représentatif pour chaque secteur: secteur nord: $\mathrm{Y} 8$; secteur central: $\mathrm{C} 4$; secteur sud: J4. Dans le cas des rongeurs, le NMI a été calculé selon le secteur à partir des premières molaires inférieures, ce qui explique l'absence dans cette figure de taxons comme Castor et Hystrix qui ne sont pas représentés par cet élément.

Fig. 3: Representation in percentages and by sector of the minimum number of individuals for taxa identified at Cueva del Camino. In order to avoid eventual biases resulting from the uneven extension of the various sectors, we have used a single representative excavation square for each sector: sector north: Y8; sector central: C4; sector south: J4. In the case of rodents, the minimum number of individuals has been calculated for each sector only taking into account the first lower molars, which explains the absence of taxa like Castor and Hystrix in this figure since they are not represented by this element in the assemblages.

\section{3 - INSECTIVORES ET CHIROPTÈRES}

Il y a sept espèces d'insectivores (Erinaceomorpha + Soricomorpha) à Cueva del Camino toutes représentées actuellement dans la Vallée du Lozoya. La taupe (Talpa europaea/occidentalis) qui domine largement (respectivement nord: $51,8 \%$; central : $80,4 \%$; et sud : $27,3 \%$; fig. 3), les musaraignes à dents rouges (Sorex gr. araneus et Sorex minutus) et la musaraigne à dents blanches
(Crocidura russula) sont représentées dans les trois secteurs. Le hérisson (Erinaceus europaeus), élément considéré comme étant plus ou moins thermophile, est présent dans le secteur nord et central mais absent du secteur sud. Enfin le crossope (Neomys fodiens/anomalus) est présent dans les secteurs nord et sud. Dans le secteur central, les restes d'insectivores sont notablement moins abondants que dans le secteur nord. Le secteur sud n'a fourni que quelques restes d'insectivores avec toutefois 
la présence de fossiles indiscutables du desman des Pyrénées (Galemys pyrenaicus).

Les chiroptères montrent une richesse spécifique selon les secteurs similaire aux insectivores. Ainsi, ont été identifiés les restes d'au moins cinq espèces différentes dans le secteur nord, deux d'entre elles actuellement absentes de la vallée: le petit rhinolophe fer à cheval (Rhinolophus hipposideros) et le murin de Natterer (Myotis nattereri), qui ne sont représentés que dans ce secteur. Le secteur central n'a fourni qu'une seule espèce (Myotis myotis/blythii) et le secteur sud a fourni quelques restes de trois espèces: Rhinolophus ferrumequinum, Myotis myotis/blythii et Plecotus auritus/austriacus.

\section{4 - RONGEURS ET LAGOMORPHES}

Les rongeurs sont le groupe le plus diversifié avec 17 espèces. Parmi celles-ci, Arvicola sapidus, A. cf. terrestris, Microtus arvalis, M. agrestis, M. gr. duodecimcostatus, Pliomys coronensis, Apodemus sylvaticus et Eliomys quercinus sont représentées dans tous les secteurs du gisement, alors que Sciurus vulgaris, Castor fiber, M. cabrerae et Clethrionomys glareolus se trouvent seulement dans les secteurs nord et central. Dans le secteur nord dominent $A$. sylvaticus $(44,6 \%)$ et $M$. gr. duodecimcostatus $(28,5 \%)$ et en plus sont représentés dans ce secteur certaines formes exclusives comme $M$. vaufreyi, $A$. bursae, $M$. cf. malei et Hystrix cf. brachyura (fig. 3). Dans le secteur central, les espèces dominantes sont $M$. gr. duodecimcostatus $(42,0 \%), M$. agrestis $(11,6 \%)$ et $M$. arvalis $(26,4 \%)$, alors que dans le secteur sud cette dernière espèce devient l'espèce dominante en représentant $60,3 \%$ de l'assemblage. Enfin, Chionomys nivalis est uniquement représenté dans le secteur sud.

En ce qui concerne les lagomorphes, le lapin (Oryctolagus cunniculus) est l'espèce la plus abondante dans tout le gisement, alors que le lièvre (Lepus sp.) est représenté par un nombre très limité de restes, ce qui empêche pour le moment de préciser son attribution au niveau de l'espèce.

\section{5 - DISCUSSION}

Un des aspects les plus marquants de l'assemblage de petits vertébrés de Cueva del Camino est, comme nous l'avons dit dans le titre, sa haute richesse spécifique, avec 49 espèces de vertébrés de petite taille comprenant micromammifères, reptiles et amphibiens. Cette haute richesse spécifique est d'autant plus frappante si on la compare avec des gisements proches et de chronologie similaire. Ainsi, le site de Preresa (Sesé et al., 2011b), situé à $70 \mathrm{~km}$ au sud de Cueva del Camino et avec un âge très proche ( $84 \pm 5,6 \mathrm{ka}$ ), présente une association de petits mammifères moins riche, constituée par 10 espèces, en comparaison des 31 espèces représentées à Cueva del Camino. Ceci peut être lié à plusieurs facteurs distincts que nous allons examiner.
En premier lieu, l'échantillonnage exhaustif et prolongé du gisement au cours des dix années de campagnes de fouilles a permis de récupérer les restes des taxons les moins fréquents présents dans le site. Durant chaque campagne, environ deux tonnes de sédiment en moyenne ont été traitées par la méthode du lavage-tamisage, ce qui suppose qu'au total, ce sont près de 20 tonnes de sédiment qui ont été lavées. Le lavage-tamisage des sédiments avec des tamis de maille fine (le plus petit ayant une maille de $0,5 \mathrm{~mm}$, suivant en ceci la proposition de Daams \& Freudenthal, 1988) conjointement avec le tri des refus à l'aide d'une loupe binoculaire a permis la récupération de la plus grande partie des dents et autres éléments osseux pour ces espèces de vertébrés de petite taille. Certaines espèces comme le castor (Castor fiber), le porc-épic (Hystrix cf. brachyura), l'écureuil roux (Sciurus vulgaris), le campagnol roussâtre (Clethrionomys glareolus), le desman des Pyrénées (Galemys pyrenaicus), le petit rhinolophe fer à cheval (Rhinolophus hipposideros) ou la rainette (Hyla sp.) ont été trouvés dans des proportions très faibles dans le gisement $(<0,1 \%)$, et un effort moindre d'échantillonnage aurait très probablement eu pour conséquence l'absence de certaines d'entre elles. D'un autre côté, certaines espèces de taille particulièrement petite, comme la musaraigne pygmée (Sorex minutus), ne peuvent être récupérées que par une telle méthodologie. Ce facteur néanmoins n'explique pas la plus grande richesse spécifique de ce gisement par rapport à d'autres où un effort similaire a été fourni pour la récupération des petits vertébrés (par exemple Preresa; Sesé et al., 2011b).

En deuxième lieu, il existe des facteurs biogéographiques qui favorisent actuellement une haute richesse spécifique en petits vertébrés dans cette région et qui, probablement, ont pu aussi agir de la même façon dans le passé. Le Système Central espagnol, de par sa position au centre de la péninsule Ibérique, fonctionne dans une certaine mesure comme une frontière entre les régions d'influence eurosibérienne situées au nord-ouest, avec un climat plus tempéré et humide, et celles d'influence méditerranéenne situées au sud-est, avec un climat plus chaud et sec (Tellería, 1999). D'un autre côté, du fait de sa plus grande élévation, les précipitations sont plus abondantes dans le Système Central que dans les territoires voisins (Agencia Estatal de Meteorología, 2011). Actuellement, la diversité et l'abondance de micromammifères dans la péninsule Ibérique montrent une corrélation positive avec les précipitations moyennes annuelles (Barbosa \& Benzal, 1996; Soriguer et al., 2003). Pour les amphibiens, les facteurs influant sur la richesse spécifique sont l'évapotranspiration actuelle annuelle (une mesure de la disponibilité conjointe d'énergie et d'eau dans un environnement) et les précipitations (Rodríguez et al., 2005; Aragón et al., 2010). C'est pourquoi certaines espèces rencontrent leur limite de distribution méridionale dans ce système montagneux, comme le desman (Galemys pyrenaicus), le campagnol des champs (Microtus arvalis), le campagnol de Male (Microtus malei), la musaraigne pygmée (Sorex minutus), le triton marbré (Triturus marmoratus), la grenouille ibérique 
(Rana iberica), l'orvet (Anguis fragilis) ou le lézard des Pyrénées (Iberolacerta monticola). Ce facteur est aussi la raison pour laquelle les gisements pléistocènes situées dans le Système Central en général, et Cueva del Camino en particulier, présentent une plus forte richesse spécifique que ceux situés plus au nord dans la Meseta castilloléonaise (par exemple Cueva de la Zarzamora; Sala et al., 2011), ou plus au sud dans le Bassin de Madrid (Sesé \& Soto, 2000, 2002a,b; Sesé et al., 2011a,b).

En troisième lieu, la localisation du gisement dans le fond d'une vallée fermée de dimensions restreintes (près de $300 \mathrm{~km}^{2}$ ), entourée de reliefs allant jusqu'à $2400 \mathrm{~m}$ d'altitude (c'est-à-dire plus de $1000 \mathrm{~m}$ de dénivelé par rapport au gisement) détermine l'existence aujourd'hui, et probablement aussi durant le début du Pléistocène supérieur, d'une grande variété d'habitats aux alentours immédiats du site adéquate pour maintenir des espèces de petits vertébrés avec des préférences écologiques distinctes. C'est pour cela, que nous trouvons dans la vallée des espèces de petits vertébrés liées à des milieux de prairies, de zones rocheuses, de tourbières, de bord de cours d'eau et de milieux forestiers, entre autres. Ce fait est d'autant plus évident lorsque nous comparons la richesse spécifique de Cueva del Camino avec d'autres gisements situés dans des vallées avec un relief plus uniforme, comme ceux du Bassin de Madrid.

Enfin, une petite partie de cette longue liste d'espèces de petits vertébrés de Cueva del Camino est due à des différences de distribution entre les différents secteurs. Néanmoins, la contribution de ce facteur à la richesse spécifique observée dans le gisement est réduite, du fait que le secteur nord contient la presque totalité des espèces présentes dans le gisement, sauf Chionomys nivalis et Galemys pyrenaicus, qui comme nous l'avons déjà dit, ne se trouvent que dans le secteur sud où, en revanche, M. cabrerae est absent. Ces différences dans la composition des associations de petits vertébrés entre secteurs distincts sont probablement autant dues à de petites différences de chronologie que de contexte climatique, entre les différents secteurs, comme le mettent en évidence les datations réalisées, qui indiquent que le secteur sud est légèrement plus moderne (environ $75 \mathrm{ka}$, MIS 5a) que les secteurs nord et central (environ $90 \mathrm{ka}$, MIS 5b). Cette explication semble être plus probable que les autres alternatives, comme celle qui suggère que ces différences soient le reflet d'une sélection introduite par les agents accumulateurs de la concentration de petits vertébrés. D'une part, la présence de $C$. nivalis dans le secteur sud est en accord avec ces datations plus récentes pour ce secteur en comparaison avec le reste du gisement. Dans la péninsule Ibérique, $C$. nivalis est en effet absent des associations de micromammifères du Pléistocène moyen et aussi de la base du Pléistocène supérieur. La citation la plus ancienne de l'espèce correspond très probablement aux niveaux $\mathrm{X}$ et $\mathrm{XI}$ de Cueva de la Carihuela (Ruiz Bustos, 2000), datés entre 70 et $80 \mathrm{ka}$ (Fernández et al., 2007), ce qui laisserait supposer que l'entrée de C. nivalis à travers les
Pyrénées se soit produite à la fin du stade isotopique marin 5 (vraisemblablement au cours du MIS 5a). Son absence dans les secteurs nord et central serait donc très probablement due à ce que leur âge soit plus ancien que l'entrée de cette espèce dans la péninsule Ibérique. D'autre part, la présence de $C$. nivalis dans le secteur sud coïncide avec l'absence de $M$. cabrerae et avec une importante réduction de la diversité dans ce secteur en relation avec une évolution climatique vers un climat plus froid dans le secteur sud que dans les autres secteurs. En effet, à l'intérieur de l'association de rongeurs du secteur sud M. arvalis est l'espèce dominante, constituant dans certains échantillons de ce secteur jusqu'à $90 \%$ de l'association de petits mammifères. Ces données suggèrent donc des différences importantes entre le milieu pendant lequel se forme le secteur sud par rapport aux autres secteurs, et renforcent l'idée que les différences quant à la représentation des espèces entre les différents secteurs soient dues plus à des facteurs chronologiques et climatiques qu'aux autres alternatives énoncées dans ce travail.

\section{6 - CONCLUSION}

Les sédiments datés du début du Pléistocène supérieur (fin du MIS 5 : MIS 5b et MIS 5a) du gisement de Cueva del Camino (Pinilla del Valle, Madrid) ont livré les restes fossiles d'au moins 49 espèces de petits vertébrés ( 7 anoures, 11 reptiles, 7 insectivores, 5 chiroptères, 17 rongeurs et 2 lagomorphes), représentant ainsi l'un des assemblages en petits vertébrés pléistocènes les plus diversifiés pour la péninsule Ibérique. Bien qu'aujourd'hui la Haute Vallée du Lozoya au centre de l'Espagne soit actuellement aussi l'un des environnements naturels les plus diversifiés en ce qui concerne les petits vertébrés terrestres dans la péninsule Ibérique, avec 13 espèces d'amphibiens, 17 de reptiles et 26 de micromammifères, les raisons de cette haute richesse spécifique au cours du Pléistocène supérieur ancien ont été mises en relation avec 1) la méthodologie de récupération systématique employée durant 10 campagnes de fouille à Cueva del Camino, 2) la localisation biogéographique du gisement à la limite entre les zones d'influence eurosibérienne et méditerranéenne et dans le Système Central espagnol avec des sommets à proximité avoisinant $2400 \mathrm{~m}$ d'altitude et occasionnant des précipitations moyennes annuelles plus élevées que dans les territoires voisins, 3 ) les particularités topographiques de la Haute Vallée du Lozoya permettant une forte hétérogénéité d'habitat ayant donc pu favoriser des espèces avec des besoins écologiques différents, et enfin 4) de petites différences chronologiques (et par conséquent probablement aussi climatiques) entre les différents secteurs qui ont alors permis d'enregistrer dans le secteur sud (très légèrement plus récent que les secteurs nord et central) l'entrée plus tardive dans la péninsule Ibérique du campagnol des neiges (Chionomys nivalis) entre 80 et $70 \mathrm{ka}$ (MIS 5a). 


\section{REMERCIEMENTS}

La Communauté de Madrid, le Ministère de la Culture, le Groupe Mahou et le Canal de Isabel II ont soutenu et financé les campagnes de fouilles ainsi que l'étude des gisements de Pinilla del Valle. Nous sommes aussi reconnaissants aux fouilleurs du Projet de Pinilla del Valle ainsi qu'aux étudiants qui ont participé au tri des petits vertébrés dans le site de fouille et au Museo Arqueológico Regional de Madrid. Cette étude est aussi une contribution aux projets de recherche CGL2012-38358 (Ministerio de Economia y Competitividad), SGR2009324 (Generalitat de Catalunya), UCM-910607 (Universidad Complutense de Madrid) et S2010/BMD-2330 (programme d'activités I+D entre groupes de recherche de la Communauté de Madrid).

\section{RÉFÉRENCES BIBLIOGRAPHIQUES}

AGENCIA ESTATAL DE METEOROLOGÍA, 2011 - Atlas climático ibérico. Instituto Nacional de Meteorología, Madrid, $79 \mathrm{p}$.

AGUIRRE E. 1989 - Vertebrados del Pleistoceno continental. In A. Pérez-González, P. Cabra Gil \& A. Martin-Serrano (coord.), Mapa del cuaternario de España: Escala 1:1.000.000. Instituto Tecnológico Geominero de España, Madrid, 47-69.

ALFÉREZ F., MOLERO G., MALdONADO E., BUSTOS V., BREA P. \& BUITRAGO, A.M., 1982 - Descubrimiento del primer yacimiento cuaternario (Riss-Würm) de vertebrados con restos humanos en la provincia de Madrid (Pinilla del Valle). Coloquios de Paleontología, 37, 15-32.

ALFÉREZ F. \& ROLDÁN B., 1992 - Un molar humano Anteneandertal con patología traumática procedente del yacimiento cuaternario de Pinilla del Valle. Munibe (Suplemento), 8, 183-188.

ARAGÓN P., LOBO J.M., OLALLA-TÁRRAGA M.Á. \& RODRÍGUEZ M.Á., 2010 - The contribution of contemporary climate to ectothermic and endothermic vertebrate distributions in a glacial refuge. Global Ecology and Biogeography, 19 (1), 40-49.

ARRIBAS A. \& JORDÁ J.F. 1999 - Los mamíferos del Cuaternario kárstico de Guadalajara (Castilla-La Mancha, España). In E. Aguirre \& I. Rábano (coord.), La huella del pasado; Fósiles de CastillaLa Mancha. Junta de Comunidades de Castilla-La Mancha, Toledo, 327-354.

ARSUAGA J.L., BAQUEDANO E., PÉREZ-GONZÁLEZ A., SALA M.T.N., GARCÍA N., ÁLVAREZ D., LAPLANA C., HUGUET R., SEVILLA P., MALDONADO E., BLAIN H.-A., QUAM R., RUIZ-ZAPATA M.B., SALA P., GIL GARCÍA M.J., UZQUIANO P. \& PANTOJA A., 2010 - El yacimiento arqueopaleontológico del Pleistoceno Superior de la Cueva del Camino en el Calvero de la Higuera (Pinilla del Valle, Madrid). Zona Arqueológica, 13, 422-442.

ARSUAGA J.L., BAQUEDANO E., PÉREZ-GONZÁlez A., SALA N., QUAM R.M., RODRÍGUEZ L., GARCÍA R., GARCÍA N., ÁLVAREZ-LAO D., LAPLANA C. HUGUET R., SEVILla P., MALDONADO E., BLAIN H.-A., RUIZZAPATA M.B., SALA P., GIL-GARCÍA M.J., UZQUIANO P., PANTOJA A. \& MÁRQUEZ B., 2012 - Understanding the ancient habitats of the last-interglacial (late MIS 5) Neanderthals of central Iberia: Paleoenvironmental and taphonomic evidence from the Cueva del Camino (Spain) site. Quaternary International, 275, 55-75.

BAQUedano E., ARSUAGa J.L. \& PÉREZ-GONZÁlez A., 2010 - Homínidos y carnívoros: competencia en un mismo nicho ecológico pleistoceno: los yacimientos del Calvero de la Higuera en Pinilla del Valle. In Consejería de Cultura y Deportes \& Comunidad de Madrid (eds.), Actas de las quintas jornadas de Patrimonio Arqueológico en la Comunidad de Madrid. Dirección General de Patrimonio Histórico, Madrid, 61-72.

BAILON S., 1991 - Amphibiens et reptiles du Pliocène et du Quaternaire de France et d'Espagne: mise en place et évolution des faunes. Thèse de Doctorat, Université Paris 7 Diderot, $499 \mathrm{p}$.

BAILON S., 1999 - Différentiation ostéologique des Anoures (Amphibia, Anura) de France. Fiches d'ostéologie animale pour l'archéologie. Série C, Varia, 1, 1-38.
BARAHONA F. \& BARBADILLO L.J., 1997 - Identification of some Iberian lacertids using skull characters. Revista Española de Herpetología, 11, 47-62.

BARAHONA QUINTANA F.F., 1996 - Osteología craneal de Lacértidos de la Península Ibérica e Islas Canarias: Análisis sistemático filogenético. Doctoral thesis, Universidad Autónoma de Madrid, Madrid, $514 \mathrm{p}$

BARBOSA A. \& BENZAL J., 1996 - Diversity and abundance of small mammals in Iberia: peninsular effect or habitat suitability? Zeitschrift für Säugertierkunde, 61 (4), 236-241.

BLAIN H.-A. 2005 - Contribution de la paléoherpétofaune (Amphibia et Squamata) à la connaissance de l'évolution du climat et du paysage du Pliocène supérieur au Pléistocène moyen d'Espagne. Thèse de Doctorat, Muséum national d'Histoire naturelle, Paris, $402 \mathrm{p}$.

CHALINE J., 1972 - Les rongeurs du Pléistocène moyen et supérieur de France: systématique, biostratigraphie, paléoclimato logie. Cahiers de Paléontologie. Éditions du Centre National de la Recherche Scientifique, Paris, $410 \mathrm{p}$

CHALINE J. (dir.), 1974 - Les Proies des rapaces : petits mammifères et leur environnement. Doin, Paris, $141 \mathrm{p}$.

DAAMS R. \& FREUDENTHAL M., 1988 - Synopsis of the DutchSpanish collaboration program in the Neogene of Calatayud-Teruel Basin. 1976-1986. Scripta Geologica, Special Issue, 1, 3-18

DÍEZ J.C., 1993 - Estudio tafonómico de los macrovertebrados de yacimientos del Pleistoceno medio. Complutum, 4, 21-40.

ESTEBAN M. \& SANCHIZ B., 1985 - Herpetofauna de Erralla. Munibe. Antropología-Arkeología, 37, 81-86.

ESTEBAN M. \& SANCHIZ B., 1991 - Sobre la presencia de Rana iberica en el Pleistoceno burgalés. Revista Española de Herpetología, 5, 93-99.

FELTEN H., HELFRICHT A. \& STORCH G., 1973 - Die Bestimmung der europäischen Fledermäuse nach der distalen Epiphyse des Humerus. Senckenbergiana Biologica, 54 (4/6), 291-297.

FERNÁNDEZ S., FUENTES N., CARRIÓN J.S., GONZÁLEZ SAMPÉRIZ P., MONTOYA E., FIL G., VEGA-TOSCANO G. \& RIQUELME J.A., 2007 - The Holocene and Upper Pleistocene pollen sequence of Carihuela Cave, southern Spain. Geobios, 40 (1), 75-90

HUGUET R., ARSUAGA J.L., PÉREZ-GONZÁLEZA., ARRIAZA M.C., SALA-BURGOS M.T.N., LAPLANA C., SEVILLA P. GARCÍA-GARCÍA N., ÁLVAREZ-LAO D., BLAIN H.-A. \& BAQUEDANO E., 2010 - Homínidos y hienas en el Calvero de la Higuera (Pinilla del Valle, Madrid) durante el Pleistoceno Superior. Resultados preliminares. Zona Arqueológica, 13, 444-458

LÓPEZ-GARCÍA J.M., 2011 - Los micromamíferos del Pleistoceno Superior de la Península Ibérica. Evolución de la diversidad taxonómica y cambios paleoambientales. Editorial Académica Española Saarbrücken, $416 \mathrm{p}$.

PALACIOS D., DE ANDRÉS N., DE MARCOS J. \& VÁZQUEZSELEM L., 2012 - Glacial landforms and their paleoclimatic significance in Sierra de Guadarrama, Central Iberian Peninsula. Geomorphology, 139-140, 67-78.

PÉREZ-GONZÁLEZ A., KARAMPAGLIDIS T., ARSUAGA J.L., BAQUEDANO E., BÁREZ S., GÓMEZ J.J., PANERA J., MÁRQUEZ B., LAPLANA C., MOSQUERA M., HUGUET R. SALA P., ARRIAZA M.C., BENITO A., ARACIL E. \& MALDONADO E., 2010 - Aproximación geomorfológica a los yacimientos del Pleistoceno Superior del Calvero de la Higuera en el Valle Alto del Lozoya (Sistema Central Español, Madrid). Zona Arqueológica, 13, 404-419.

PRIETO CANA D. \& DE LUCIO J.V., 1995 - Especies y hábitats del Valle Alto del Lozoya: conocimiento actual sobre su estado de conservación. Serie Documentos, 17. Centro de Investigación de Espacios Naturales Protegidos Fernando González Bernáldez, Soto del Real, 1-88.

RODRÍGUEZ M.Á., BELMONTES J.A. \& HAWKINS B.A., 2005 - Energy, water and large-scale patterns of reptile and amphibian species richness in Europe. Acta Oecologica, 28 (1), 65-70.

RUIZ BUSTOS A., 2000 - Estudio paleoecológico de los sedimentos con presencia del hombre de Neandertal en la Cueva de la Carihuela (Piñar, Granada). Ayuntamiento de Píñar, Granada, 91 p.

SALA M.T.N., ARSUAGA J.L., LAPLANA C., RUIZ ZAPATA B. GIL GARCÍA M.J., GARCÍA N., ARANBURU A. \& ALGABA M., 2011 - Un paisaje de la Meseta durante el Pleistoceno Superior. Aspectos paleontológicos de la Cueva de la Zarzamora (Segovia, España). Boletín de la Real Sociedad Española de Historia Natural. Sección Geológica, 105 (1-4), 67-85.

SESÉ C. \& SEVILLA P., 1996 - Los micromamíferos del Cuaternario peninsular español: Cronoestratigrafía e implicaciones bioestratigráficas. Revista Española de Paleontología, no extraordinario 278-287. 
SESÉ C. \& SOTO E., 2000 - Vertebrados del Pleistoceno de Madrid. Arqueología, Paleontología y Etnografía, 6, 216-243.

SESÉ C. \& SOTO E., 2002a - Vertebrados del Pleistoceno del Jarama y Manzanares. Zona Arqueológica, 1, 319-337.

SESÉ C. \& SOTO E., 2002b - Catálogo de los yacimientos de vertebrados del Pleistoceno en las terrazas de los ríos Jarama y Manzanares. Zona Arqueológica, 1, 431-457.

SESÉ C., PANERA J., RUBIO-JARA S. \& PÉREZ-GONZÁleZ A., 2011a - Micromamíferos del Pleistoceno medio y Pleistoceno Superior en el Valle del Jarama: yacimientos de Valdocarros y HAT (Madrid, España). Estudios Geológicos, 67 (1), 131-151.

SESÉ C., RUBIO-JARA S., PANERA J. \& PÉREZ-GONZÁleZ A., 2011b - Micromamíferos del Pleistoceno Superior del Yacimiento de PRERESA en el Valle del Manzanares y su contribución a la reconstrucción paleoambiental de la Cuenca de Madrid durante el Pleistoceno. Estudios Geologicos, 67 (2), 471-494.

SEVILLA P., 1988 - Estudio paleontológico de los Quirópteros del Cuaternario español. Paleontologia i Evolució, 22, 113-233.
SORIGUER R.C., CARRO F., MÁRQUEZ F.J. \& FANDOS P. 2003 - La diversidad y abundancia de los micromamíferos ibéricos. In J.M. Pérez Jiménez (coord.), In memoriam al Prof. Dr. Isidoro Ruiz Martínez. Universidad de Jaén, Servicio de Publicaciones e Intercambio Científico, Jaén, 439-478.

SOTO E. \& SESÉ C., 1987 - Mamíferos del Pleistoceno del municipio de Madrid. Estudios de Prehistoria y Arqueología Madrileñas, 5, 11-35.

SZYNDLAR Z., 1984 - Fossil snakes from Poland. Acta Zoologica Cracoviensia, 28, 1-156.

TELLERÍA J.L., 1999 - La diversidad de vertebrados del Valle de El Paular (Madrid). In Servicio de Conservación y Protección del Medio Natural (coord.), Primeros Encuentros Científicos del Parque Natural de Peñalara y del Valle de El Paular. El Paular (Rascafría): Mayo 1998. Consejería de Medio Ambiente, Comunidad de Madrid, Guadarrama, 155-162. 